University of Nebraska - Lincoln

DigitalCommons@University of Nebraska - Lincoln

Michigan Bovine Tuberculosis Bibliography and

Database

Wildlife Disease and Zoonotics

2007

\title{
Vaccination of White-Tailed Deer (Odocoileus virginianus) with Mycobacterium bovis Bacillus Calmette Guerin
}

\author{
M. V. Palmer \\ United States Department of Agriculture, Agricultural Research Service, National Animal Disease Center, \\ Mitchell.Palmer@ars.usda.gov \\ T. C. Thacker \\ United States Department of Agriculture, Agricultural Research Service, National Animal Disease Cente \\ W. R. Waters \\ United States Department of Agriculture, Agricultural Research Service, National Animal Disease Center
}

Follow this and additional works at: https://digitalcommons.unl.edu/michbovinetb

Part of the Veterinary Medicine Commons

Palmer, M. V.; Thacker, T. C.; and Waters, W. R., "Vaccination of White-Tailed Deer (Odocoileus virginianus) with Mycobacterium bovis Bacillus Calmette Guerin" (2007). Michigan Bovine Tuberculosis Bibliography and Database. 108.

https://digitalcommons.unl.edu/michbovinetb/108

This Article is brought to you for free and open access by the Wildlife Disease and Zoonotics at DigitalCommons@University of Nebraska - Lincoln. It has been accepted for inclusion in Michigan Bovine Tuberculosis Bibliography and Database by an authorized administrator of DigitalCommons@University of Nebraska Lincoln. 


\title{
Vaccination of white-tailed deer (Odocoileus virginianus) with Mycobacterium bovis bacillus Calmette Guerín
}

\author{
M.V. Palmer*, T.C. Thacker, W.R. Waters \\ United States Department of Agriculture, Agricultural Research Service, National Animal Disease Center, \\ 2300 Dayton Avenue, Ames, IA 50010, USA
}

Received 6 April 2007; received in revised form 12 June 2007; accepted 26 June 2007

Available online 17 July 2007

\begin{abstract}
Wildlife reservoirs of Mycobacterium bovis represent serious obstacles to the eradication of tuberculosis in domestic livestock. In Michigan, USA tuberculous white-tailed deer transmit $M$. bovis to cattle. One approach in dealing with this wildlife reservoir is to vaccinate deer in order to interrupt the cycle of deer to deer and deer to cattle transmission. Thirty-one white-tailed deer were assigned to one of three groups; 2 SC doses of $10^{7} \mathrm{CFU}$ of $M$. bovis BCG $(n=11)$; $1 \mathrm{SC}$ dose of $10^{7} \mathrm{CFU}$ of $M$. bovis BCG $(n=10)$; or unvaccinated deer $(n=10)$. After vaccination, deer were inoculated intratonsilarly with $300 \mathrm{CFU}$ of virulent $M$. bovis. Gross lesion severity scores of the medial retropharyngeal lymph node were significantly reduced in deer receiving 2 doses of BCG compared to unvaccinated deer. Vaccinated deer had fewer lymph node granulomas than unvaccinated deer, and most notably, fewer late stage granulomas characterized by coalescent caseonecrotic granulomas containing numerous acid-fast bacilli. BCG was isolated from 7/21 vaccinated deer as long as 249 days after vaccination. In one case BCG was transmitted from a vaccinated deer to an unvaccinated deer. In white-tailed deer BCG provides measurable protection against challenge with virulent $M$. bovis. However, persistence of vaccine within tissues as well as shedding of BCG from vaccinates remain areas for further investigation.
\end{abstract}

Published by Elsevier Ltd.

Keywords: BCG; Deer; Mycobacterium bovis; Tuberculosis; Vaccination

\section{Introduction}

In 1994, a free-ranging white-tailed deer (Odocoileus virginianus) in Michigan was diagnosed with tuberculosis due to Mycobacterium bovis, the causative agent of bovine tuberculosis [1]. Subsequent surveys identified a focus of M. bovis infection in free-ranging white-tailed deer in northeast Michigan [2,3]. This represents the first known reservoir of M. bovis in free-ranging wildlife in the United States and a significant obstacle to the eradication of bovine tuberculosis in domestic livestock. Several factors are thought to have contributed to the establishment and persistence of $M$. bovis in this wildlife reservoir. It is postulated that $M$. bovis was transmitted from cattle to deer at some time during the mid 1900s when a

\footnotetext{
* Corresponding author. Tel.: +1 515663 7474; fax: +1 5156637458 .

E-mail address: Mitchell.Palmer@ars.usda.gov (M.V. Palmer).
}

large number of Michigan cattle were infected with M. bovis [4]. During this same period, Michigan's deer population was steadily increasing beyond normal habitat carrying capacity to focal concentrations of $19-23 \mathrm{deer} / \mathrm{km}^{2}$ [1]. Transmission and maintenance of $M$. bovis among deer is thought to have been facilitated by the common practice in Michigan of longterm winter feeding of deer by private citizens to prevent migration and decrease winter mortality in order to keep deer numbers high for hunting purposes [1].

Current disease control measures in Michigan include decreasing deer density through increased hunting and strict control of feeding and baiting of white-tailed deer. Another possible control measure could be vaccination of white-tailed deer to prevent infection, disease, or transmission. Vaccination of captive farmed red deer in New Zealand has shown that vaccination with $M$. bovis BCG can prevent infection and disease (i.e. lesion development) [5,6]. BCG vaccination 
has also been used to control tuberculosis in farmed sika deer (Cervus nippon) in China [7].

M. bovis BCG was first used as an anti-tuberculosis vaccine in humans in 1921 and is one of the oldest and most widely used vaccines in the world today [8]. Although reports of efficacy in humans vary widely by geographic region and among different age groups, consistent protection has been observed against miliary tuberculosis and tuberculous meningitis when used as a single dose in neonates. The great variability in efficacy is often attributed to one or more factors including, differences in vaccines strains, prior host sensitization to various non-tuberculous environmental mycobacteria, genetic differences in trial populations, or trial design [8].

Protection against $M$. bovis or M. tuberculosis has been shown to be associated with a TH1 immune response; generally characterized by production of cytokines such as IFN- $\gamma$, IL-12, and TNF- $\alpha$. It would appear then that any successful vaccine against tuberculosis would require the induction of a TH1 immune response. The immune response to $M$. bovis BCG has been investigated in white-tailed deer [9]. Upon stimulation with $M$. bovis PPD, CD4+ and $\gamma / \delta \mathrm{T}$ cells proliferated in vaccinated but not unvaccinated deer. Likewise, peripheral blood mononuclear cells (PBMC) from vaccinated but not unvaccinated deer produced IFN- $\gamma$ upon stimulation with $M$. bovis PPD [9]. However, the ability of vaccination with $M$. bovis BCG to protect against infection or disease in white-tailed deer has not been explored. The purpose of the current study was to determine the ability of parenterally administered $M$. bovis BCG to prevent infection or disease in white-tailed deer after intratonsilar challenge with virulent M. bovis.

\section{Materials and methods}

\subsection{Animals and bacteria}

Thirty-one white-tailed deer $(\sim 1$ year old $)$ were obtained from a captive breeding herd (tuberculosis- and paratuberculosis-free) at the National Animal Disease Center (Ames, IA, USA). All deer were housed and cared for according to institutional guidelines. Deer were randomly assigned to one of three groups; $2 \mathrm{SC}$ doses of $10^{7}$ colony-forming units (CFU) of M. bovis BCG administered 6 weeks apart $(n=11)$; $1 \mathrm{SC}$ dose of $10^{7} \mathrm{CFU}$ of M. bovis BCG $(n=10)$; or unvaccinated deer $(n=10)$. The M. bovis BCG (Pasteur strain) as well as the challenge strain M. bovis 1315 were grown in Middlebrook's 7H9 media supplemented with 10\% oleic acid-albumin-dextrose complex (Difco, Detroit, MI) plus $0.05 \%$ Tween 80 (Sigma Chemical Co., St. Louis, MO) as described [10]. Mid log-phase growth bacilli were pelleted by centrifugation at $750 \times g$, washed twice with phosphate buffered saline (PBS) (0.01 M, pH 7.2), and diluted to the appropriate cell density in $2 \mathrm{ml}$ of PBS. Bacilli were enumerated by serial dilution plate counting on Middlebrook's 7H11 selective media (Becton Dickinson, Cockeysville, MD). A single vaccine dose consisted of $10^{7} \mathrm{CFU} M$. bovis $\mathrm{BCG}$ in $1.5 \mathrm{ml} \mathrm{PBS}$ and was administered SC. Individual vaccine doses were thoroughly vortexed immediately prior to injection to disperse bacilli in suspension and minimize clumping. In the 2 dose group a 6-week interval separated doses. Unvaccinated and vaccinated deer were housed together in an outdoor paddock prior to challenge with virulent M. bovis, at which time they were moved to appropriate biosecurity level 3 (BL-3) animal housing.

Seventy-seven days after the 2 dose group received the second dose of vaccine all deer were inoculated intratonsilarly with approximately $150 \mathrm{CFU}$ of $M$. bovis strain 1315 into each tonsilar crypt for a total dose of $300 \mathrm{CFU}$, as described previously [11]. Strain 1315 was originally isolated from a white-tailed deer in Michigan. For inoculation deer were anesthetized by IM injection of a combination of xylazine $(2 \mathrm{mg} / \mathrm{kg}$ ) (Mobay Corporation, Shawnee, KS) and ketamine $(6 \mathrm{mg} / \mathrm{kg}$ ) (Fort Dodge Laboratories, Fort Dodge, IA). After inoculation the effects of xylazine were reversed by IV injection of tolazoline $(4 \mathrm{mg} / \mathrm{kg}$ ) (Lloyd Laboratories, Shanandoah, IA). Animals were housed in BL-3 housing, and fed a commercial pelleted feed with free access to water. All procedures were approved by the National Animal Disease Center Institutional Animal Care and Use Committee prior to the beginning of the experiment.

\subsection{Interferon- $\gamma$ enzyme-linked immunosorbent assay}

A whole-blood culture system for the determination of recall IFN- $\gamma$ production as used for cattle [12] and red deer [13] was adapted for use with samples from white-tailed deer [14]. Briefly, $1.5 \mathrm{ml}$ heparinized blood was added to 24-well tissue culture plates. Treatments included $100 \mu \mathrm{l}$ PBS (i.e., no stimulation), $20 \mu \mathrm{g} / \mathrm{ml} M$. bovis purified protein derivative (PPD), $20 \mu \mathrm{g} / \mathrm{ml} \mathrm{M}$. avium PPD, $10 \mu \mathrm{g} / \mathrm{ml}$ rESAT-6:CFP-10, or $20 \mu \mathrm{g} / \mathrm{ml}$ pokeweed mitogen (PWM). Optimal dilutions of PPDs, recombinant proteins and PWM were determined previously [14]. Samples were incubated for $48 \mathrm{~h}$ at $37^{\circ} \mathrm{C}$ in a humidified chamber with $5 \% \mathrm{CO}_{2}$. Samples were then centrifuged $(400 \times g)$, and plasma was harvested and stored at $-80^{\circ} \mathrm{C}$ until analyzed for IFN- $\gamma$ by enzymelinked immunosorbent assay (ELISA) with a commercially available kit $\left(\right.$ Cervigam $^{\mathrm{TM}}$, Prionics AG, Schlieren, Switzerland). Interferon- $\gamma$ concentrations $(\mathrm{ng} / \mathrm{ml})$ in test samples were determined by comparing the absorbance of test samples with the absorbance of standards within a linear curve fit.

\subsection{ELISA for antibody to lipoarabinomannan (LAM)-enriched mycobacterial antigen preparations}

Antigens were prepared from M. bovis strain 1315 and antibody measured as described [15]. Bacilli were harvested from 4-week cultures, sonicated in PBS, further disrupted with $0.1-0.15 \mathrm{~mm}$ diameter glass beads (Biospec Products, Bartlesville, OK) in a bead beater (Biospec Products), cen- 
trifuged, filtered $(0.22 \mu \mathrm{m}$ pore size $)$, and digested in a $1-\mathrm{mg} / \mathrm{ml}$ proteinase $\mathrm{K}$ (Roche Molecular Biochemicals, Indianapolis, IN) solution (50 M Tris, $1 \mathrm{mM} \mathrm{CaCl}_{2}$ buffer, $\mathrm{pH}$ 8.0) for $1 \mathrm{~h}$ at $50^{\circ} \mathrm{C}$. Protein concentrations were determined (BioRad, Hercules, CA) and antigens were stored at $-20^{\circ} \mathrm{C}$. The antigen concentration used for ELISA was $40 \mu \mathrm{g} / \mathrm{ml}$.

Immulon II 96 well microtiter plates (Dynatech, Chantilly, VA) were coated with $100-\mu 1 /$ well antigen diluted in carbonate/bicarbonate coating buffer ( $\mathrm{pH}$ 9.6). Antigen coated plates, including control wells containing coating buffer alone, were incubated for $15 \mathrm{~h}$ at $4{ }^{\circ} \mathrm{C}$. Plates were washed three times with $200 \mu \mathrm{l} /$ well containing $0.05 \%$ Tween 20 (i.e., PBST; Sigma), and blocked with $200 \mu \mathrm{l} /$ well commercial milk diluent/blocking solution (Kirkegaard and Perry Laboratories, Gaithersburg, MD). After incubation for $1 \mathrm{~h}$ at $37^{\circ} \mathrm{C}$ in the blocking solution, wells were washed nine times with $200 \mu \mathrm{l} /$ well PBST and test sera added to wells (100 $\mu \mathrm{l} /$ well). Test and control sera were determined by evaluation of the reactivity of twofold serial dilutions ranging from 1:6 to 1:800 (volume of sera/volume of diluent ratio) with antigen. After incubation for $20 \mathrm{~h}$ at $4{ }^{\circ} \mathrm{C}$ with diluted test sera, wells were washed nine times with $200 \mu \mathrm{l} /$ well PBST and incubated for $1 \mathrm{~h}$ at $37^{\circ} \mathrm{C}$ with $100 \mu \mathrm{l} /$ well horseradish peroxidase (HRP)conjugated anticervine immunoglobulin $\mathrm{G}$ (IgG) heavy and light chains (Kirkegaard and Perry) diluted 1:500 in PBS plus $0.1 \%$ gelatin. Wells were washed nine times with $200 \mu \mathrm{l} / \mathrm{well}$ PBST and incubated for $4.5 \mathrm{~min}$ at room temperature with $100 \mu \mathrm{l} /$ well 3,3',5,5'-tetramethylbenzidine. The reaction was stopped by addition of $100 \mu \mathrm{l} /$ well $0.18 \mathrm{M}$ sulfuric acid, and the A450 of individual wells was measured with an automated ELISA plate reader (Molecular Devices, Menlo Park, $\mathrm{CA})$. Changes in optical density readings $(\Delta \mathrm{OD})$ were calculated by subtracting the mean OD readings for wells receiving coating buffer alone (two replicates) from the mean OD readings for antigen coated wells (two replicates) receiving the same serum sample.

\subsection{Necropsy and tissue sampling}

One hundred and thirty days after challenge with virulent $M$. bovis all deer were euthanized by IV sodium pentobarbital. At necropsy, the following tissues or fluids were collected and processed for isolation of $M$. bovis and microscopic analysis as described [16]; palatine tonsil, lung, liver, mandibular, parotid, medial retropharyngeal, tracheobronchial, mediastinal, hepatic, mesenteric and prefemoral lymph nodes. Tissues were processed for isolation of $M$. bovis as previously described [17]. Isolates of $M$. bovis were identified by colony morphology, growth, and biochemical characteristics as well as by PCR.

Lymph nodes were cross-sectioned at $0.5 \mathrm{~cm}$ intervals and examined. Each lung lobe was examined separately and cross-sectioned at $0.5-1.0 \mathrm{~cm}$ intervals. Lungs and lymph nodes were subjected to semi-quantitative scoring of gross lesions adapted from Vordermeier et al. [18]. Lung lobes (left cranial, left caudal, right cranial, right caudal, middle and accessory) were subjected to the following scoring system: (0) no visible lesions; (1) no external gross lesions, but lesions seen upon slicing; (2) $<5$ gross lesions of $<10 \mathrm{~mm}$ in diameter; (3) $>5$ gross lesions of $<10 \mathrm{~mm}$ in diameter; (4) $>1$ distinct gross lesion of $>10 \mathrm{~mm}$ in diameter; (5) coalescing gross lesions. Scoring of lymph node gross lesions was based on the following scoring system: (0) no visible lesions; (1) small focal lesion (1-2 $\mathrm{mm}$ in diameter); (2) several small foci; (3) extensive lesions. Tissues collected for microscopic analysis were fixed by immersion in $10 \%$ neutral buffered formalin and included all tissues collected for bacteriologic examination. For microscopic examination, formalin-fixed tissues were processed by routine paraffin-embedment techniques, cut in $5 \mu \mathrm{m}$ sections and stained with hematoxylin and eosin (HE). Adjacent sections were cut from samples containing lesions suggestive of tuberculosis (caseonecrotic granulomata) and stained by the Ziehl-Neelsen technique for identification of acid-fast bacteria (AFB). Numbers of AFB were recorded for each granuloma. Due to an inability to reliably enumerate large numbers of AFB, granulomas with $>200$ AFB were recorded as $>200$ AFB. Microscopic tuberculous lesions were staged (I-IV) according to criteria adapted from that described by Rhoades et al. [19]. Stage I (initial) granulomas were characterized by accumulations of epithelioid macrophages with low numbers of lymphocytes and neutrophils. Multinucleated giant cells may be present but necrosis was absent. Acidfast bacilli, when present, were seen within macrophages or multinucleated giant cells. Stage II (solid) granulomas were characterized by accumulations of epithelioid macrophages surrounded by a thin connective tissue capsule. Infiltrates of neutrophils and lymphocytes may be present as well as multinucleated giant cells. Necrosis when present was minimal. Stage III (necrotic) granulomas were characterized by complete fibrous encapsulation. Necrotic cores were surrounded by a zone of epithelioid macrophages admixed with multinucleated giant cells and lymphocytes. Stage IV (necrotic and mineralized) granulomas were characterized by a thick fibrous capsule surrounding irregular multicentric granulomas with multiple necrotic cores. Necrotic cores contained foci of dystrophic mineralization. Epithelioid macrophages and multinucleated giant cells surrounded necrotic areas and there were often moderate to marked infiltrates of lymphocytes. Acid-fast bacilli were often present in moderate numbers and primarily located within the caseum of the necrotic core.

Additionally, each animal was assigned to one of three classifications according to the scheme by Griffin et al. [6]: (1) uninfected animals with no gross or microscopic lesions and from which $M$. bovis could not be isolated by bacteriological culture from samples collected, (2) infected animals from which $M$. bovis could be isolated by bacteriological culture, but no gross or microscopic lesions were present, and (3) diseased animals with gross or microscopic lesions consistent with tuberculosis and from which $M$. bovis could be isolated by bacteriological culture. 


\subsection{PCR methods}

PCR was used to confirm M. bovis infection and to distinguish M. bovis from M. bovis BCG Pasteur. Region of difference 1 (RD1), while present in virulent $M$. bovis is absent from M. bovis BCG [20]. The following primer pairs were used to detect the presence or absence of the RD1 region using a method similar to that described by Talbot et al. [21]; RD1-1 GTTCATGCTCGCGGACTAC, RD1-2 ATACATCGGTGACCCTTTGC and RD1-3 TAGGTTCGGTTACGCTGGTT. Primers RD1-1 and RD1-3 produce a 542 bp product from $M$. bovis BCG Pasteur while RD-2 and RD-3 produce a 384 bp product from $M$. bovis.

Mycobacteria were transferred to $25 \mu l$ of sterile TE using a sterile-disposable inoculation loop. The bacterial suspension was heat inactivated by heating to $80^{\circ} \mathrm{C}$ for $10 \mathrm{~min}$, vortexed, then heated for an additional $10 \mathrm{~min}$. One microliter of the bacterial preparation was added to the following PCR master mix: $1 \times$ PCR reaction buffer with $\mathrm{MgCl}_{2}$ (Roche Applied Science), $50 \mathrm{pM}$ of each primer, $200 \mu \mathrm{M}$ of each dNTP (PCR Nucleotide Mix, Roche Applied Science), 2 U FastStart Taq (Roche Applied Science), $5 \mu \mathrm{g}$ BSA (Ambion, Austin, TX) in a total reaction volume of $50 \mu$ l. Touchdown PCR was performed with an initial preheating step of $2 \mathrm{~min}$ at $94{ }^{\circ} \mathrm{C}$ followed by denaturation at $94^{\circ} \mathrm{C}$ for $45 \mathrm{~s}$, annealing started at $65^{\circ} \mathrm{C}$ for $1 \mathrm{~min}$ with a $1^{\circ} \mathrm{C}$ decrease per cycle followed by extension at $72^{\circ} \mathrm{C}$ for $2 \mathrm{~min}$, after the annealing temperature reached $50^{\circ} \mathrm{C}$ an additional 29 cycles were performed. A final extension at $72{ }^{\circ} \mathrm{C}$ for 10 min was included. Samples were analyzed by electrophoresis on $1.5 \%$ agarose gel and visualized with ethidium bromide.

Isolates of acid-fast bacteria that were not identified by PCR as virulent $M$. bovis or M. bovis BCG Pasteur were further identified using 16S ribosomal DNA sequencing as described previously [22]. Sequences were then identified through the use of a mycobacterial species sequence database [23].

\subsection{Statistical analysis}

Mean group values for lesion scores were compared using one-way repeated measures analysis of variance (GraphPad Prism, GraphPad Software, San Diego, CA). Differences between means were then compared using the Bonferonni method. Results of IFN- $\gamma$ analysis and ELISA were compared using one-way analysis of variance followed by Tukey-Kramer multiple comparisons test using a commercially available statistics program (InStat 2.00, GraphPad Software). A $p$-value $<0.05$ was considered significant.

\section{Results}

Regardless of group the most common site for lesion development was the medial retropharyngeal lymph node (9 of 10 diseased deer). Pulmonary lesions were less com-
Table 1

Summary of gross lesions, microscopic lesions and bacteriological isolation of Mycobacterium bovis from white-tailed deer vaccinated with $M$. bovis BCG Pasteur and challenged by intratonsilar inoculation of $300 \mathrm{CFU}$ of virulent $M$. bovis

\begin{tabular}{|c|c|c|c|}
\hline & Unvaccinated & $\begin{array}{l}\text { BCG } 1 \\
\text { dose }\end{array}$ & $\begin{array}{l}\text { BCG } 2 \\
\text { doses }\end{array}$ \\
\hline Gross lesions & $5 / 10$ & $2 / 10$ & $1 / 11$ \\
\hline Microscopic lesions & $6 / 10$ & $4 / 10$ & $3 / 11$ \\
\hline Isolation of virulent $M$. bovis & $5 / 10$ & $3 / 10$ & $5 / 11$ \\
\hline Isolation of $M$. bovis $\mathrm{BCG}$ & $1 / 10$ & $4 / 10$ & $3 / 11$ \\
\hline $\begin{array}{l}\text { Isolation of } M \text {. bovis } \mathrm{BCG} \text { from } \\
\text { tissues with lesions }\end{array}$ & $0 / 1$ & $0 / 4$ & $2 / 3^{\mathrm{a}}$ \\
\hline $\begin{array}{l}\text { Isolation of } M \text {. bovis } \mathrm{BCG} \text { only } \\
\text { and not virulent } M \text {. bovis }\end{array}$ & $1 / 10$ & $4 / 10$ & $2 / 11$ \\
\hline $\begin{array}{l}\text { Isolation of non-tuberculous } \\
\text { mycobacteria }\end{array}$ & $1 / 10^{\mathrm{b}}$ & $2 / 10^{\mathrm{b}}$ & $1 / 11^{\mathrm{c}}$ \\
\hline $\begin{array}{l}\text { Deer from which virulent } M \text {. } \\
\text { bovis was not isolated and } \\
\text { lesions were not seen }\end{array}$ & $4 / 10$ & $7 / 10$ & $6 / 11$ \\
\hline
\end{tabular}

mon, being present in 4 of 10 diseased deer. Six of $10(60 \%)$ unvaccinated deer developed gross or microscopic lesions consistent with tuberculosis (Table 1) and would be categorized as diseased while $2 / 10(20 \%)$ and $2 / 11(18 \%)$ of $M$. bovis $\mathrm{BCG}$ vaccinated deer were categorized as diseased in the 1 dose and 2 dose groups, respectively.

Gross lesion severity scores of the medial retropharyngeal lymph node were significantly reduced in deer receiving 2 doses of $M$. bovis BCG compared to unvaccinated deer (Fig. 1) but not significantly different compared to deer receiving a single dose of BCG. Lesion severity scores of the lungs did not differ between groups (data not shown). Total pathology scores (sum of scores for lymph nodes and lung) were lower ( $p=0.1$ ) in deer receiving 2 doses of BCG (Fig. 2) compared to unvaccinated deer or deer receiving a single dose of BCG. Microscopic evaluation of the medial retropharyngeal lymph nodes revealed a greater number of granulomas in unvaccinated deer than in vaccinated deer (Table 2). All deer, regardless of vaccination status, had microscopic lesions in all stages. However, vaccinated deer had fewer stage IV granulomas than unvaccinated deer. Enumeration of AFB within granulomas revealed that stage IV granulomas had a mean of $>200 \mathrm{AFB}$ /granuloma while those of stages I-III had a mean of 0-11 AFB/granuloma (Table 3).

Table 2

Numbers of granulomas at different microscopic stages of development ${ }^{\mathrm{a}}$ in the medial retropharyngeal lymph node of white-tailed deer vaccinated with M. bovis BCG and challenged by intratonsilar inoculation of $300 \mathrm{CFU}$ of virulent $M$. bovis

\begin{tabular}{lccclc}
\hline & Stage I & Stage II & Stage III & Stage IV & Total \\
\hline BCG 2 doses & 2 & 3 & 5 & 1 & 11 \\
BCG 1 dose & 1 & 4 & 3 & 2 & 10 \\
Unvaccinated & 18 & 18 & 11 & 7 & 54 \\
\hline
\end{tabular}

${ }^{\text {a }}$ Stages defined in text. 


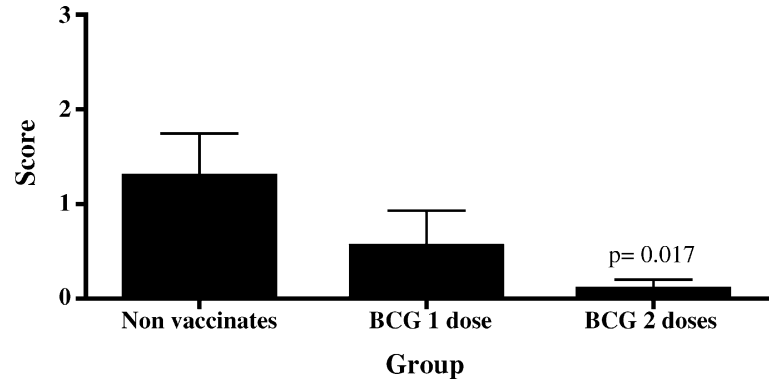

Fig. 1. Gross lesion severity scores from medial retropharyngeal lymph nodes of unvaccinated deer $(n=10)$ or deer vaccinated SC with one $(n=10)$ or two doses $(n=11)$ of $10^{7} \mathrm{CFU}$ Mycobacterium bovis BCG Pasteur and challenged intratonsilarly with $300 \mathrm{CFU}$ of virulent $M$. bovis. Scores represent the mean \pm standard error. Scoring system as follows: (0) no visible lesions; (1) small focal lesion (1-2 mm in diameter); (2) several small foci; (3) extensive lesions.

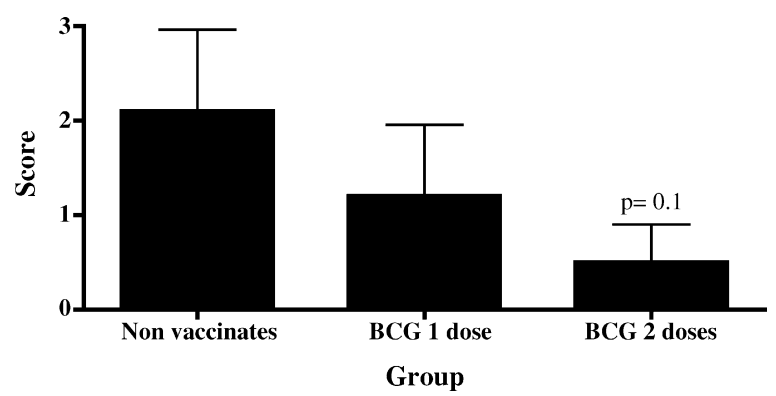

Fig. 2. Total combined gross lesion severity scores from lymph nodes and lungs of unvaccinated deer $(n=10)$ or deer vaccinated SC with one $(n=10)$ or two doses $(n=11)$ of $10^{7} \mathrm{CFU}$ M. bovis BCG Pasteur and challenged intratonsilarly with $300 \mathrm{CFU}$ of virulent $M$. bovis. Scores represent the mean \pm standard error. Scoring system as in Fig. 1 for lymph nodes. Lung scoring system as follows: (0) no visible lesions; (1) no external gross lesions, but lesions seen upon slicing; (2) $<5$ gross lesions of $<10 \mathrm{~mm}$ in diameter; (3) $>5$ gross lesions of $<10 \mathrm{~mm}$ in diameter; (4) $>1$ distinct gross lesion of $>10 \mathrm{~mm}$ in diameter; (5) coalescing gross lesions.

Virulent $M$. bovis was isolated from 4 deer with no gross or microscopic lesions (i.e. infected deer). Infected, but not diseased deer included 1 deer from the 1 dose group and 3 deer from the 2 dose group. Uninfected deer (deer from which virulent $M$. bovis could not be recovered and in which no gross or microscopic lesions were seen) included 4 unvaccinated deer, 7 deer from the 1 dose group and 6 deer from the 2 dose group (Table 1).

Table 3

Mean number of AFB in medial retropharyngeal lymph node granulomas of different developmental stages ${ }^{\text {a }}$ from white-tailed deer vaccinated with M. bovis BCG and challenged by intratonsilar inoculation of $300 \mathrm{CFU}$ of virulent $M$. bovis

\begin{tabular}{lllcc}
\hline & Stage I & Stage II & Stage III & Stage IV \\
\hline BCG 2 doses & 1 & 0 & 5.4 & $>200^{\mathrm{b}}$ \\
BCG 1 dose & 0 & 0 & 11.3 & $>200$ \\
Unvaccinated & 0 & 0.17 & 0.18 & $>200$ \\
\hline
\end{tabular}

${ }^{\text {a }}$ Stages defined in text.

b Due to an inability to reliably enumerate large numbers of AFB, granulomas with $>200 \mathrm{AFB}$ were recorded as $>200 \mathrm{AFB}$.
M. bovis BCG was isolated at the time of necropsy from 8 deer, including 1 unvaccinated deer, 4 deer from the 1 dose group and 3 deer from the 2 dose group. Isolations of BCG were made from various tissues including, medial retropharyngeal, tracheobronchial, mediastinal and hepatic lymph nodes. $M$. bovis BCG was isolated from a single site in 5 deer, 2 sites in 2 deer and 3 sites in 1 deer. In 2 deer the isolation of BCG was made from tissues (hepatic and mediastinal lymph nodes) in which microscopic, but not gross lesions, were seen. In one of these 2 deer, virulent $M$. bovis was also isolated from a microscopic lesion in the hepatic lymph node. The mediastinal lymph node was the only tissue from which BCG was isolated from the single unvaccinated deer. In addition to virulent $M$. bovis and $M$. bovis BCG, $M$. terrae and $M$. kansasii were isolated from 4 deer. In all cases M. terrae was isolated from tissues not containing gross or microscopic lesions. However, M. kansasii was isolated from a mediastinal lymph node in which gross and microscopic lesions were seen. Within that same animal, other tissues contained lesions from which virulent $M$. bovis was isolated.

Prior to challenge with virulent $M$. bovis, vaccinated deer but not unvaccinated deer demonstrated IFN- $\gamma$ production in response to M. bovis PPD stimulation (Fig. 3A). Although not statistically significant, deer receiving 2 doses of BCG had higher levels of IFN- $\gamma$ production after booster vaccination, than did deer receiving 1 dose of BCG. After intratonsilar challenge with virulent $M$. bovis both vaccinated and unvaccinated deer were shown to produce IFN- $\gamma$ upon stimulation with $M$. bovis PPD.

When rESAT-6:CFP-10 was used in place of $M$. bovis PPD, neither vaccinated nor unvaccinated deer demonstrated IFN- $\gamma$ production prior to challenge with virulent $M$. bovis (Fig. 3B). Similar to findings with $M$. bovis PPD, IFN- $\gamma$ production was evident in all groups in response to rESAT6:CFP-10 after challenge with virulent $M$. bovis. However, in contrast to responses seen upon stimulation with $M$. bovis PPD, IFN- $\gamma$ production in response to rESAT-6:CFP-10 was transient as levels returned to pre-challenge values by 80 days after challenge (220 days after vaccination). Prior to initiation of the study all 3 deer from which $M$. terrae was isolated did not show IFN- $\gamma$ responses to $M$. bovis PPD, $M$. avium PPD or rESAT-6:CFP-10. Following challenge, IFN- $\gamma$ responses were moderate to $M$. bovis PPD and rESAT-6:CFP10 compared to other deer in the same groups. The single deer from which M. kansasii was isolated demonstrated moderate IFN- $\gamma$ responses to $M$. bovis PPD, M. avium PPD and rESAT6:CFP-10 prior to the study as well as following vaccination and challenge.

Antibody responses to a LAM-enriched M. bovis antigen were greater in vaccinated deer than in unvaccinated deer (Fig. 4). Optical density values were significantly greater in deer receiving 2 doses of BCG 63 and 112 and days after primary vaccination compared to unvaccinated deer or deer receiving a single dose of BCG. A significant boost in anti- 


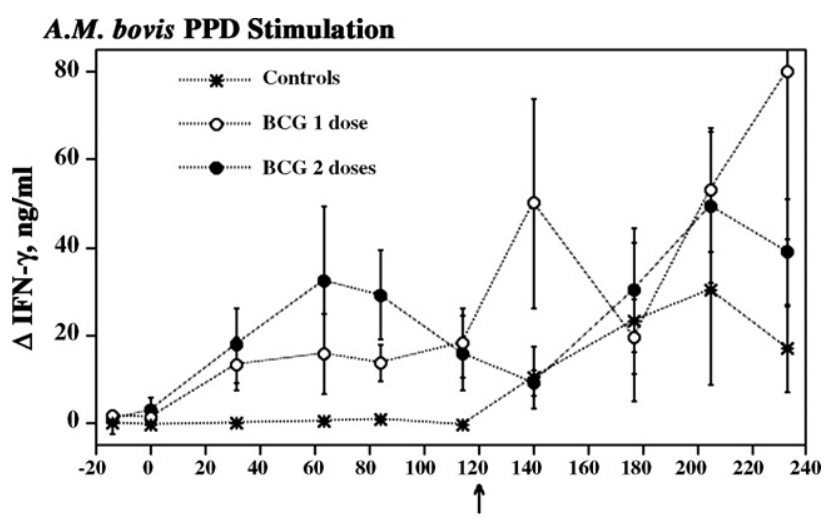

B. rESA T-6:CFP10 Stimulation

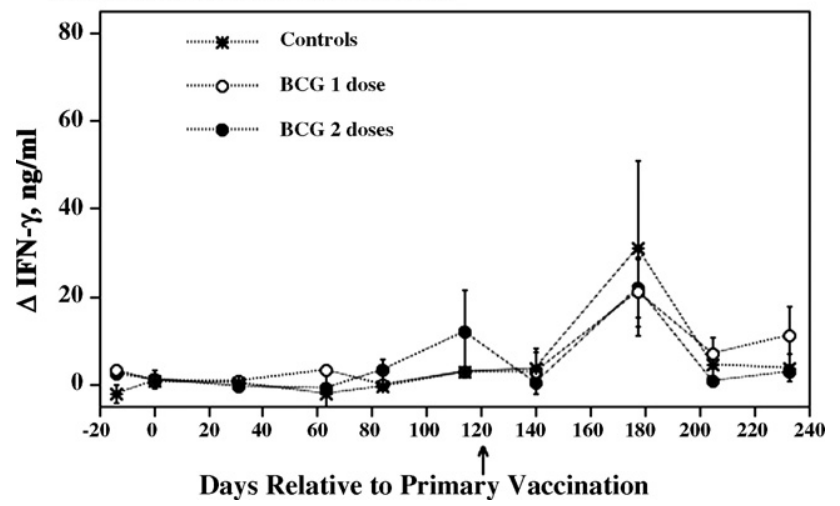

Fig. 3. Interferon- $\gamma$ production in response to stimulation with $M$. bovis PPD (A) or rESAT-6:CFP10 (B) as measured by optical density (OD, $450 \mathrm{~nm}$ ). Interferon- $\gamma$ concentrations $(\mathrm{ng} / \mathrm{ml})$ in test samples were determined by comparing the absorbance of test samples with the absorbance of standards within a linear curve fit. Samples from unvaccinated deer $(n=10)$ or deer vaccinated with one $(n=10)$ or two doses $(n=11)$ of $10^{7} \mathrm{CFU}$ of $M$. bovis BCG were analyzed for antigen specific IFN- $\gamma$ production prior to and after vaccination (day 0) and challenge with $300 \mathrm{CFU}$ of virulent M. bovis (arrow, day 119). Background OD values (stimulation with PBS alone) have been subtracted from the response to $M$. bovis PPD or rESAT-6:CFP10. Data are represented as means \pm standard error.

body was not seen in vaccinated deer after challenge with virulent $M$. bovis.

\section{Discussion}

Previous studies in red deer established that doses of approximately $5 \times 10^{4}$ to $5 \times 10^{7} \mathrm{CFU}$ of $M$. bovis BCG (Pasteur) provided protection against challenge with virulent $M$. bovis $[5,6]$. These studies used intratonsilar challenge doses of 100-500 CFU of virulent $M$. bovis similar to those used in the present study. Also similar to findings in the current study, two doses of BCG provided superior protection in red deer compared to a single dose [6]. Although a single dose of M. bovis BCG did not protect red deer against infection, it did result in fewer animals with tuberculous lesions. The present study design was patterned after extensive studies in red deer that used a similar BCG strain with a similar interval between priming and boosting doses of vaccine as well as a similar interval between challenge and necropsy [5]. In those studies

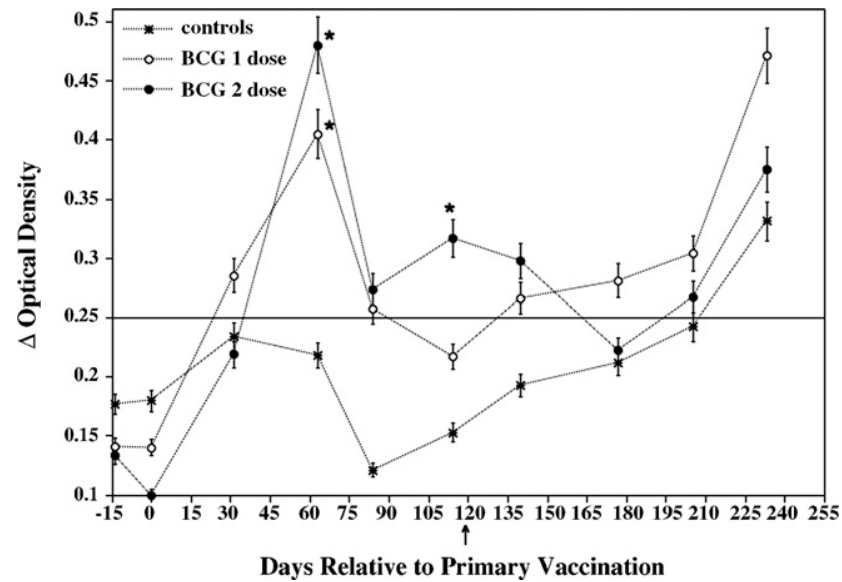

Fig. 4. Response kinetics of serum antibody specific for LAM-enriched antigen. Sera from unvaccinated deer $(n=10)$ or deer vaccinated with one $(n=10)$ or two doses $(n=11)$ of $10^{7} \mathrm{CFU}$ of $M$. bovis BCG was analyzed for reactivity to $M$. bovis derived LAM by ELISA prior to and after vaccination (day 0) and challenge with $300 \mathrm{CFU}$ of virulent M. bovis (arrow, day 119). Data are represented as means \pm standard error. *Significantly different from unvaccinated deer $(p<0.05)$.

the interval from boosting dose to challenge and the interval from challenge to necropsy did not decrease the protective effect of vaccination. In contrast, the interval between priming and boosting doses of vaccine was critical as prolonged intervals (i.e. 43 weeks) resulted in decreased protection [5]. The current findings are also in agreement with vaccination studies in cattle, where vaccination with a similar dose of $M$. bovis BCG (Pasteur) followed by intratracheal challenge with virulent $M$. bovis resulted in less severe lesions in vaccinated cattle compared to unvaccinated cattle [24]. In a separate study, cattle dosed twice with $M$. bovis BCG demonstrated fewer granulomas, less necrosis and fewer acid-fast bacilli than unvaccinated cattle [25]. Studies to date in deer, and most studies in cattle, have used BCG strain Pasteur. It is unknown if other strains of BCG would differ in protective efficacy in white-tailed deer compared to BCG Pasteur. Recently both BCG strains Pasteur and Danish were reported to provide similar levels of protection in vaccinated calves [26].

Similar to previous findings in white-tailed deer and red deer, IFN- $\gamma$ production in response to $M$. bovis PPD was evident in vaccinated, but not unvaccinated deer. After vaccination, no significant differences were seen in deer receiving 1 dose of $M$. bovis BCG compared to deer receiving 2 doses. IFN- $\gamma$ production was not detected in vaccinated deer prior to challenge when rESAT-6:CFP-10 was used as antigen instead of M. bovis PPD. However, IFN- $\gamma$ responses to rESAT-6:CFP-10 responses were robust in all deer after challenge with virulent $M$. bovis, suggesting that responses to rESAT-6:CFP-10 could prove useful in differentiating BCG vaccinated from $M$. bovis infected deer. The lack of response to rESAT-6:CFP-10 is due to the absence of the RD1 region of the genome in $M$. bovis BCG compared to virulent $M$. bovis. The genes responsible for ESAT-6 and CFP-10 production are located within this RD1 region. The protein ESAT-6 
is co-secreted by members of the M. tuberculosis complex in a tight 1:1 complex with CFP-10 [27]. Genes for both ESAT- 6 and CFP-10 are absent in many non-tuberculous mycobacteria as well as the vaccine M. bovis BCG. However, esat-6 and $c f p-10$ are present in a subset of non-tuberculous mycobacteria such as $M$. kansasii, M. marinum, M. leprae and M. smegmatis [28-30]. The presence of esat-6 and cfp10 in M. kansasii, likely explains the pre-vaccination and pre-challenge response to rESAT-6:CFP-10 seen in the deer from which $M$. kansasii was isolated. Lack of response to these recombinant antigens by BCG vaccinated animals has been proposed as a means of differentiating vaccinated from infected animals [31,32] as well as differentiating $M$. bovis infected animals from those infected with other mycobacterial pathogens such as M. avium subsp. paratuberculosis [33]. Lipoarabinomannan is a cell wall glycolipid of mycobacteria. LAM-based ELISA assays, similar to that used in the present study, have demonstrated utility for diagnosis of mycobacterial diseases including bovine tuberculosis and paratuberculosis $[34,35]$.

Although the present study is the first to examine the efficacy of BCG vaccination of white-tailed deer and demonstrate some protection against challenge with virulent $M$. bovis, successful vaccination of wildlife poses various challenges. It is doubtful that parenteral vaccination, such as that used in the present study, would be feasible with free-ranging wildlife on a large scale. More likely, an oral vaccine such as that currently used to vaccinate wildlife against rabies would be necessary [36]. Oral delivery of live BCG; while posing several challenges, has been shown to be feasible with white-tailed deer [37]. Oral or intragastric vaccination of brushtail possums (Trichosurus vulpecula) with $M$. bovis BCG produces inferior protection to intraduodenal vaccination [38-40]. However, lipid based preparations to protect live M. bovis BCG from the harmful effects of the gastric environment have proven effective in brushtail possum vaccination trials [41].

In red deer, $M$. bovis BCG Pasteur was still present in tissues from the site of injection as well as the draining lymph nodes 3 months after SC vaccination with $2 \times 10^{6} \mathrm{CFU}$ of M. bovis BCG Pasteur [42]. Gross lesions in regional lymph nodes and transmission from vaccinated deer to unvaccinated deer have not been features of studies involving red deer [43] or cattle. In the current study, unlike red deer, BCG not only persisted in vaccinated deer, but spread to multiple sites, was associated with microscopic granulomas, and in one case, was shed by vaccinated deer and transmitted to a unvaccinated deer, being recovered from the mediastinal lymph node. Studies in mice demonstrate persistence of $M$. bovis BCG for up to 30 weeks and spread to distant organs after SC vaccination $[44,45]$. Murine studies further suggest that persistence of $M$. bovis BCG within a vaccinated host is vital in sustaining long-lasting immunological memory. Vaccinated mice receiving chemotherapy to eliminate residual post-vaccinal BCG demonstrated inferior cell-mediated immune responses and inferior protection against challenge with virulent $M$. tuberculosis as measured by colonization of the spleen by $M$. tuberculosis when compared to mice still harboring low numbers of BCG [45,46]. Interestingly, persistence of BCG has not been a feature of human vaccination studies. Even among individuals with symptomatic AIDS, bacteremia following BCG immunization is reported to be uncommon [47]. However, recent analysis shows the risk of disseminated disease from BCG vaccination may be significantly greater in HIV infected infants compared to uninfected infants [48].

Isolation of non-tuberculous mycobacteria from several deer illustrates the challenges posed by exposure to saprophytic non-tuberculous mycobacteria present in the environment. Although exposure of red deer to environmental saprophytic mycobacteria did not influence susceptibility to experimental infection with $M$. bovis [6], the low number of deer similarly exposed in the present study precludes any definitive conclusions; however, all 3 deer from which $M$. terrae was isolated were categorized as uninfected while the single deer from which $M$. kansasii was isolated was categorized as diseased. It is unclear from the current study whether exposure to saprophytic environmental mycobacteria had a positive or negative effect on vaccine efficacy. Studies in cattle have shown that sensitization of calves to environmental saprophytic mycobacteria adversely affects the protective efficacy of BCG vaccination [49]. Likewise, one explanation for the highly variable, and often disappointing, efficacy observed in human vaccine trials has been exposure to saprophytic environmental mycobacteria [50]. Vaccination of neonatal calves has been used as a strategy to avoid prior sensitization by saprophytic environmental mycobacteria. Indeed, vaccination of neonatal calves induces a higher level of immunity than that seen in calves vaccinated at 5-6 months of age [44,51-53]. A similar strategy could be feasible for vaccination of captive white-tailed deer, but would be problematic for vaccination of free-ranging white-tailed deer. Although the ultimate goal of any tuberculosis vaccination program is to eradicate tuberculosis, a more immediate aim is to reduce the rate of transmission between susceptible hosts. Any tuberculosis vaccine considered for use in free-ranging white-tailed deer may not need to protect animals against infection. Disease prevalence could be decreased if vaccinated animals did not develop advanced lesions or shed virulent $M$. bovis, thereby decreasing disease transmission. The present study demonstrates that parenteral vaccination of white-tailed deer with M. bovis BCG Pasteur provides some protection against challenge with virulent $M$. bovis. The present study also reveals several points that will require further investigation, not least of which are those related to safety. Further studies will need to investigate the virulence of $M$. bovis BCG in white-tailed deer and the degree and duration of vaccine persistence within tissues. Shedding of vaccine to other deer or non-target species, including humans, also remains in question. 


\section{Acknowledgements}

The authors thank Jay Steffen, Doug Ewing, and Todd Holtz for animal care and Bart Olthoff, Jessica Pollock, Rachel Huegel, Mike Howard and Shelly Zimmerman for technical assistance. Mention of trade names or commercial products in this article is solely for the purpose of providing specific information and does not imply recommendation or endorsement by the U.S. Department of Agriculture. This project was funded in part by Veterinary Services, Animal and Plant Health Inspection Service, USDA.

\section{References}

[1] Schmitt SM, Fitzgerald SD, Cooley TM, Bruning-Fann CS, Sullivan $\mathrm{L}$, Berry D, et al. Bovine tuberculosis in free-ranging white-tailed deer from Michigan. J Wildl Dis 1997;33(4):749-58.

[2] O’Brien DJ, Fitzgerald SD, Lyon TJ, Butler KL, Fierke JS, Clarke KR, et al. Tuberculous lesions in free-ranging white-tailed deer in Michigan. J Wildl Dis 2001;37(3):608-13.

[3] O'Brien DJ, Schmitt SM, Fierke JS, Hogle SA, Winterstein SR, Cooley TM, et al. Epidemiology of Mycobacterium bovis in free-ranging whitetailed deer, Michigan, USA, 1995-2000. Prev Vet Med 2002;54(1):47.

[4] Frye GH. Bovine tuberculosis eradication: the program in the United States. In: Thoen CO, Steele JH, editors. Mycobacterium bovis infection in animals and humans. Ames: Iowa State University Press; 1995. p. 119-29.

[5] Griffin JFT, Mackintosh CG, Rodgers CR. Factors influencing the protective efficacy of a BCG homologous prime-boost vaccination regime against tuberculosis. Vaccine 2006;24(6):835-45.

[6] Griffin JF, Mackintosh CG, Slobbe L, Thomson AJ, Buchan GS. Vaccine protocols to optimise the protective efficacy of BCG. Tuber Lung Dis 1999;79(3):135-43.

[7] Shilang Z, Shanzhi W. Prevention of tuberculosis in sika deer (Cervus nippon). Biol Deer Prod: Roy Soc N Z 1985:154.

[8] Fine PE. The BCG story: lessons from the past and implications for the future. Rev Infect Dis 1989;11(Suppl 2):S353-9.

[9] Waters WR, Palmer MV, Whipple DL, Slaughter RE, Jones SL. Immune responses of white-tailed deer (Odocoileus virginianus) to Mycobacterium bovis BCG vaccination. J Wildl Dis 2004;40(1):66-78.

[10] Bolin CA, Whipple DL, Khanna KV, Risdahl JM, Peterson PK, Molitor TW. Infection of swine with Mycobacterium bovis as a model of human tuberculosis. J Infect Dis 1997;176(6):1559-66.

[11] Palmer MV, Whipple DL, Olsen SC. Development of a model of natural infection with Mycobacterium bovis in white-tailed deer. J Wildl Dis 1999;35(3):450-7.

[12] Wood PR, Rothel JS. In vitro immunodiagnostic assays for bovine tuberculosis. Vet Microbiol 1994;40(1/2):125-35.

[13] Slobbe L, Lockhart E, Kelly J, Buchan G. The production and biological assessment of cervine interferon gamma. Cytokine 2000;12(8):1211-7.

[14] Palmer MV, Waters WR, Whipple DL, Slaughter RE, Jones SL. Evaluation of an in vitro blood-based assay to detect production of interferon-gamma by Mycobacterium bovis-infected white-tailed deer (Odocoileus virginianus). J Vet Diagn Invest 2004;16(1):17-21.

[15] Waters WR, Palmer MV, Bannantine JP, Whipple DL, Greenwald R, Esfandiari J, et al. Antigen recognition by serum antibodies in whitetailed deer (Odocoileus virginianus) experimentally infected with Mycobacterium bovis. Clin Diagn Lab Immunol 2004;11(5):849-55.

[16] Palmer MV, Waters WR, Whipple DL. Lesion development in whitetailed deer (Odocoileus virginianus) experimentally infected with Mycobacterium bovis. Vet Pathol 2002;39(3):334-40.

[17] Palmer MV, Waters WR, Whipple DL. Susceptibility of raccoons (Procyon lotor) to infection with Mycobacterium bovis. J Wildl Dis 2002;38(2):266-74.
[18] Vordermeier HM, Chambers MA, Cockle PJ, Whelan AO, Simmons J, Hewinson RG. Correlation of ESAT-6-specific gamma interferon production with pathology in cattle following Mycobacterium bovis BCG vaccination against experimental bovine tuberculosis. Infect Immun 2002;70(6):3026-32.

[19] Rhoades ER, Frank AA, Orme IM. Progression of chronic pulmonary tuberculosis in mice aerogenically infected with virulent Mycobacterium tuberculosis. Tuber Lung Dis 1997;78(1):57-66.

[20] Pym AS, Brodin P, Brosch R, Huerre M, Cole ST. Loss of RD1 contributed to the attenuation of the live tuberculosis vaccines Mycobacterium bovis BCG and Mycobacterium microti. Mol Microbiol 2002;46(3):709-17.

[21] Talbot EA, Williams DL, Frothingham R. PCR identification of Mycobacterium bovis BCG. J Clin Microbiol 1997;35(3):566-9.

[22] Kierschner P, Bottger EC. Species identification of Mycobacteria using rDNA sequencing. In: Parish T, Stoker NG, editors. Methods in molecular biology, mycobacteria protocols. Totowa, NJ: Humana Press Inc.; 1998. p. 349-61.

[23] Harmsen D, Dostal S, Roth A, Niemann S, Rothganger J, Sammeth $\mathrm{M}$, et al. RIDOM: comprehensive and public sequence database for identification of Mycobacterium species. BMC Infect Dis 2003;11(3): 26.

[24] Buddle BM, de Lisle GW, Pfeffer A, Aldwell FE. Immunological responses and protection against Mycobacterium bovis in calves vaccinated with a low dose of BCG. Vaccine 1995;13(12):1123-30.

[25] Johnson L, Gough J, Spencer Y, Hewinson G, Vordermeier M, Wangoo A. Immunohistochemical markers augment evaluation of vaccine efficacy and disease severity in bacillus Calmette-Guerin (BCG) vaccinated cattle challenged with Mycobacterium bovis. Vet Immunol Immunopathol 2006;111(3/4):219-29.

[26] Buddle BM, Wedlock DN, Denis M. Progress in the development of tuberculosis vaccines for cattle and wildlife. Vet Microbiol 2006;112(2-4):191-200.

[27] Renshaw PS, Panagiotidou P, Whelan A, Gordon SV, Hewinson RG, Williamson RA, et al. Conclusive evidence that the major Tcell antigens of the Mycobacterium tuberculosis complex ESAT-6 and CFP-10 form a tight, 1:1 complex and characterization of the structural properties of ESAT-6, CFP-10, and the ESAT- $6 *$ CFP-10 complex. Implications for pathogenesis and virulence. J Biol Chem 2002;277(24):21598-603.

[28] Geluk A, van Meijgaarden KE, Franken KL, Subronto YW, Wieles B, Arend SM, et al. Identification and characterization of the ESAT-6 homologue of Mycobacterium leprae and T-cell cross-reactivity with Mycobacterium tuberculosis. Infect Immun 2002;70(5):2544-8.

[29] Geluk A, van Meijgaarden KE, Franken KL, Wieles B, Arend SM, Faber WR, et al. Immunological crossreactivity of the Mycobacterium leprae CFP-10 with its homologue in Mycobacterium tuberculosis. Scand J Immunol 2004;59(1):66-70.

[30] Gey Van Pittius NC, Gamieldien J, Hide W, Brown GD, Siezen RJ, Beyers AD. The ESAT-6 gene cluster of Mycobacterium tuberculosis and other high $\mathrm{G}+\mathrm{C}$ Gram-positive bacteria. Genome Biol 2001;2(10):44.1-18.

[31] Buddle BM, Parlane NA, Keen DL, Aldwell FE, Pollock JM, Lightbody $\mathrm{K}$, et al. Differentiation between Mycobacterium bovis BCG-vaccinated and $M$. bovis-infected cattle by using recombinant mycobacterial antigens. Clin Diagn Lab Immunol 1999;6(1):1-5.

[32] Vordermeier HM, Cockle PC, Whelan A, Rhodes S, Palmer N, Bakker $\mathrm{D}$, et al. Development of diagnostic reagents to differentiate between Mycobacterium bovis BCG vaccination and $M$. bovis infection in cattle. Clin Diagn Lab Immunol 1999;6(5):675-82.

[33] Waters WR, Nonnecke BJ, Palmer MV, Robbe-Austermann S, Bannantine JP, Stabel JR, et al. Use of recombinant ESAT-6:CFP-10 fusion protein for differentiation of infections of cattle by Mycobacterium bovis and by $M$. avium subsp. avium and M. avium subsp. paratuberculosis. Clin Diagn Lab Immunol 2004;11(4):729-35.

[34] Waters WR, Miller JM, Palmer MV, Stabel JR, Jones DE, Koistinen KA, et al. Early induction of humoral and cellular immune responses during 
experimental Mycobacterium avium subsp. paratuberculosis infection of calves. Infect Immun 2003;71(9):5130-8.

[35] Gaborick CM, Salman MD, Ellis RP, Triantis J. Evaluation of a fiveantigen ELISA for diagnosis of tuberculosis in cattle and Cervidae. J Am Vet Med Assoc 1996;209(5):962-6.

[36] Desmettre P, Languet B, Chappuis G, Brochier B, Thomas I, Lecocq JP, et al. Use of vaccinia rabies recombinant for oral vaccination of wildlife. Vet Microbiol 1990;23(1-4):227-36.

[37] Miller LA, Johns BE, Elias DJ, Killian GJ. Oral vaccination of whitetailed deer using a recombinant Bacillus Calmette-Guerin vaccine expressing the Borrelia burgdorferi outer surface protein A: prospects for immunocontraception. Am J Reprod Immunol 1999;41(4):279-85.

[38] Aldwell FE, Keen DL, Stent VC, Thomson A, Yates GF, de Lisle GW, et al. Route of BCG administration in possums affects protection against bovine tuberculosis. N Z Vet J 1995;43(7):356-9.

[39] Aldwell FE, Pfeffer A, DeLisle GW, Jowett G, Heslop J, Keen D, et al. Effectiveness of BCG vaccination in protecting possums against bovine tuberculosis. Res Vet Sci 1995;58(1):90-5.

[40] Buddle BM, Aldwell FE, Keen DL, Parlane NA, Yates G, de Lisle GW. Intraduodenal vaccination of brushtail possums with bacille Calmette-Guerin enhances immune responses and protection against Mycobacterium bovis infection. Int J Tuberc Lung Dis 1997;1(4):377-83.

[41] Aldwell FE, Keen DL, Parlane NA, Skinner MA, de Lisle GW, Buddle BM. Oral vaccination with Mycobacterium bovis BCG in a lipid formulation induces resistance to pulmonary tuberculosis in brushtail possums. Vaccine 2003;22(1):70.

[42] Slobbe L, Lockhart E, O'Donnell MA, MacKintosh C, De Lisle G, Buchan G. An in vivo comparison of bacillus CalmetteGuerin (BCG) and cytokine-secreting BCG vaccines. Immunology 1999;96(4):517-23.

[43] Griffin JF, Hesketh JB, Mackintosh CG, Shi YE, Buchan GS. BCG vaccination in deer: distinctions between delayed type hypersensitivity and laboratory parameters of immunity. Immunol Cell Biol 1993;71(Pt 6):559-70.

[44] Aldwell FE, Cross ML, Fitzpatrick CE, Lambeth MR, de Lisle GW, Buddle BM. Oral delivery of lipid-encapsulated Mycobacterium bovis BCG extends survival of the bacillus in vivo and induces a long-term protective immune response against tuberculosis. Vaccine 2006;24(12):2071-8.

[45] Olsen AW, Brandt L, Agger EM, van Pinxteren LA, Andersen P. The influence of remaining live BCG organisms in vaccinated mice on the maintenance of immunity to tuberculosis. Scand J Immunol 2004;60(3):273-7.

[46] Cross ML, Lambeth MR, Coughlan Y, Aldwell FE. Oral vaccination of mice with lipid-encapsulated Mycobacterium bovis BCG: effect of reducing or eliminating BCG load on cell-mediated immunity. Vaccine 2007;25(7):1297-303.

[47] Waddell RD, Lishimpi K, von Reyn CF, Chintu C, Baboo KS, Kreiswirth B, et al. Bacteremia due to Mycobacterium tuberculosis or M. bovis, Bacille Calmette-Guerin (BCG) among HIV-positive children and adults in Zambia. Aids 2001;15(1):55-60.

[48] Hesseling AC, Marais BJ, Gie RP, Schaaf HS, Fine PE, GodfreyFaussett $\mathrm{P}$, et al. The risk of disseminated Bacille Calmette-Guerin (BCG) disease in HIV-infected children. Vaccine 2007;25(1):14-8.

[49] Buddle BM, Wards BJ, Aldwell FE, Collins DM, de Lisle GW. Influence of sensitisation to environmental mycobacteria on subsequent vaccination against bovine tuberculosis. Vaccine 2002;20(7/8): 1126.

[50] Brandt L, Feino Cunha J, Weinreich Olsen A, Chilima B, Hirsch P, Appelberg R, et al. Failure of the Mycobacterium bovis BCG vaccine: some species of environmental mycobacteria block multiplication of BCG and induction of protective immunity to tuberculosis. Infect Immun 2002;70(2):672-8.

[51] Buddle BM, Pollock JM, Skinner MA, Wedlock DN. Development of vaccines to control bovine tuberculosis in cattle and relationship to vaccine development for other intracellular pathogens. Int J Parasitol 2003;33(5/6):555-66.

[52] Buddle BM, Wedlock DN, Parlane NA, Corner LA, De Lisle GW, Skinner MA. Revaccination of neonatal calves with Mycobacterium bovis $\mathrm{BCG}$ reduces the level of protection against bovine tuberculosis induced by a single vaccination. Infect Immun 2003;71(11):6411-9.

[53] Hope JC, Thom ML, Villarreal-Ramos B, Vordermeier HM, Hewinson RG, Howard CJ. Vaccination of neonatal calves with Mycobacterium bovis $\mathrm{BCG}$ induces protection against intranasal challenge with virulent M. bovis. Clin Exp Immunol 2005;139(1):48-56. 